\title{
Der Neo-Lamarckismus
}

und seine

\section{Beziehungen zum Darwinismus.}

\section{Vortrag}

gehalten

in der allgemeinen Sitzung der 74. Versammlung

deutscher Naturforscher und Aerzte

in Karlsbad am 26. Sept. 1902,

mit Anmerkungen und Zusätzen herausgegeben

VOII

\section{Dr. Richard von Wettstein,} Professor an der Universität Wien.

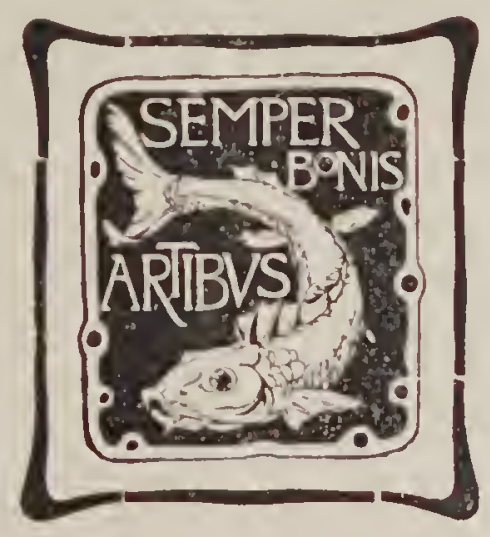

Verlag von Gustav Fischer in Jena. 1903. 
Alle Rechte vorbehalten. 


\section{Einleitung.}

Die folgenden Blätter enthalten den Wortlaut des ron mir in der allgemeinen Sitzung der 74 . Versammlung deutscher Nitturforscher und Aerzte in Karlsbad am 26. September d. J. gehaltenen Vortrages, ergänzt durch einzelne Zusätze und insbesondere durch Anmerkungen mit den notwendigen Iitteraturnachweisen. Meine Ausführungen decken sich zum Teile mit jenen des Vortrages, welchen ich in der feierlichen Sitzung der Kaiserl. Akademie der Wissenschaften in Wien am 28. Nai d. J. hielt und dessen Inhalt ich hiermit weiteren Kreisen ron Fachkollegen leichter zugänglich machen will.

Wien, im Oktober r 902.

Prof. Dr. R. v. Wettstein. 
Digitized by the Internet Archive in 2020 with funding from University of Toronto 
Es war ein glücklicher (iedanke, gelegentlich der vorjährigen Versammlung deutscher Naturforscher und Nerzte in Hamburg, der ersten im neuen Jahrhundert, in einer Reihe zusammenfassender Vorträge einen Ueberblick zu geben über den der\%eitigen Stand unserer Kenntnisse in Bezug auf jenes Problem, welches der Naturwissenschaft des vorigen Jahrhunderts die Signatur verlieh: in Bezug auf das entwickelungsgeschichtliche Problem. Schon in der vorhergehenden Versammlung in Aachen hatte O. Hertwig in einem glänzenden Vortrage über die Geschichte der Biologie im 19. Jahrhundert überhaupt die Bedeutung dieses Problemes dargelegt $\left.{ }^{1}\right)$.

Von zoologischer"), botanischer") und paläontologischer") Seite erhielten wir im vergangenen Jahre lichtrolle Referate, deren übereinstimmendes Resultat dahin lauten konnte, dass als wesentlichstes Ergebnis der biologischen Forschungen des vorigen Jahrhunderts angesehen werden kann, dass die Ueber\%eugung von dem entwickelungsgeschichtlichen Zusammenhang (ler Organismen Gemeingutallernaturwissenschaftlich D) cnkenden geworden ist. Die descendenztheoretische Auffassung allor biologischen Fragen, welche zu Beginn des r g. Jahrhunderts nur von einzelnen, ihrer Zeit vorauseilenden Forschern vertreten wurde, ist heute die Basis unserer ganzen Arbeitsmethode, und wenn wir Genugthuung über die Schaffung dieser Basis empfinden, so beruht dies nicht auf der Freude über den Sieg einer uns lieb gewordenen Idee - denn zu oft haben wir schon sehen müssen, wie siegreiche Ideen denn doch über kur\% oder lang wieder ihre Bedeutung verloren - , sondern darauf, dass die verschiedensten 
Arbeitsrichtungen und Arbeitsmethoden unabhängig voneinander und manchmal gegen ihren Willen zu dem übereinstimmenden Resultate der descendenztheoretischen Auffassung führten, worin wir wobl einen objektiven Beweis ihrer Berechtigung erblicken können.

Wenn ich mir erlaube, heute, an die Vorträge der vorigen Versammlung anknüpfend, ein einschlägiges Thema zu behandeln, so geschieht es, weil jene Vorträge nicht nur gewissermassen einen Abschluss in Bezug auf das angegebene Resultat markieren, sondern weil sie zugleich ein Programm für die Zukunft ergeben, insofern aus ihnen klar hervorgeht, dass wir Naturforscher und Aerzte wohl in der Anerkennung der Descendenzlehre einig sind, dass aber die Frage nach der Art und Weise des Zustandekommens neuer Formen, auf dem ja die Möglichkeit jeder Fortentwickelung beruht, noch keine definitive Beantwortung gefunden hat. Und darum sei es mir gestattet, hier eine Anschauung mit Nachdruck zu vertreten, die im Vorjahre von biologischer Seite keine eingehendere Begründung gefunden hat, die gerade in jüngster Zeit wieder Gegenstand energischer Angriffe war ${ }^{5}$ ).

Als ein unzweifelhaftes Ergebnis der Forschungen des letzten Jahrhunderts kann wohl auch die Ueberzeugung bezeichnet werden, dass biologische Phänomene überhaupt nicht so leicht zu erklären sind, als man lange Zeit glaubte; nicht nur die Mannigfaltigkeit der Form lässt das Arbeitsgebiet der Biologie als ein so unermessliches erscheinen, sondern auch die Mannigfaltigkeit der Vorgänge, deren Wechselwirkung in jener Formenfülle zum Ausdrucke kommt. Immer mehr wird klar, dass wir, auf dem sicheren Boden der Descendenztheorie stehend, nun erst daran gehen müssen, von Fall zu Fall die einzelnen Erscheinungen, in welchen das Problem der Entwickelung uns entgegentritt, in exalkter Weise zu prüfen, bevor wir ein abschliessendes Urteil über das Wesen der entwickelungsgeschichtlichen Vorgänge abgeben können. Von dieser Ueberzeugung ausgehend, erscheinen 
uns die bisher aufgestellten Lehren über die Entstehung neuer Formen im Tier- und Pflanzenreiche nicht so sehr als der Abschluss von Forschungsrichtungen, sondern vielmehr als mehr oder minder umfassend begründete Thesen, deren Prüfung im einzelnen erst erfolgen muss. Diese Prüfung sehe ich als einen wesentlichen Bestandteil des naturwissenschaftlichen Programmes der nächsten \%eit an ").

Von diesem Standpunkte aus wollen meine folgenden Ausführungen beurteilt werden; indem ich diesen Standpunkt einnehme, wird es verständlich sein, wenn ich hier als Botaniker spreche, mich bei Behandlung meines Themas auf Tatsachen stützte, die ich beurteilen zu können glaube.

Die I.ehren, welche bisher aufgestellt wurden zu dem \%wecke, um das Phänomen der Neubildung ron Formen, also der Artenbildung, verständlich zu machen, lassen sich bekanntlich in zwei Gruppen einordnen; in eine solche von I.ehren, welche dem Organismus die Fähigkeit zuschreiben, direkt jene Veränderungen zu erfahren, welche die obwaltenden Verhältnisse als zweckmässig erscheinen lassen und in solche. welche neue Eigentümlichkeiten des Organismus auf zufällige Aenderungen und deren Zweckmässigkeit auf das Eingreifen einer Zuchtwahl zurückführen. welche entweder das Passende fördert oder wenigstens das nicht Passende beseitigt. Lehren der ersteren Art nennen wir bekanntlich lamarckistische nach ihrem ersten Vertreter Jean Lamarck; Lehren der letzteren Art werden darwinistisch genannt. Zu den wichtigsten Ausgestaltungen des Lamarckis$\mathrm{mus}^{7}$ ) zähle ich die Lehren von H. Spencers), Eimer"'), Naegeli ${ }^{10}$ ) und Kassowitz ${ }^{11}$ ), zu den wichtigsten auf darwinistischer Anschau ung ${ }^{12}$ ) fussenden Lehren möchte ich die der Zoologen Kölliker ${ }^{13}$ ), Roux ${ }^{11}$ ) und Weismann ${ }^{15}$ ), der Botaniker A. Kerner ${ }^{16}$ ), Korschinsky ${ }^{17}$ ) und H. de Vries ${ }^{18}$ ) zählen. Es kann hier nicht meine Aufgabe sein. die Unterschiede zwischen diesen Anschauungen ausführlicher darzulegen; 
ich möchte nur erwähnen, dass es psychologisch ganz verständlich ist, wenn die Vertreter dieser Lehren vielfach in schroffsten Gegensatz zu einander traten, dass aber gerade in jüngster Zeit in gewissem Sinne eine gegenseitige Annäherung der Ansichten zu bemerken ist, indem immer mehr die Anschauung Anhänger findet, dass es überhaupt nicht möglich ist, a 1le Vorgänge der Formneubildung auf dieselbe Art zu erklären, dass lamarckistische und darwinistische Anschaungen sich nicht ausschliessen, sondern nebeneinander ihre Berechtigung haben ${ }^{19}$ ). Darwin selbst hat die teilweise Gültigk eit des Lamarckismus durchaus nicht geleugnet, $H$. Spencer hat mit klarer Begründung die Anwendbarkeit beider Lehren vertreten und nicht mehr klein ist die Zahl moderner Formen, welche denselben Standpunkt einnehmen.

Ich selbst bin von der Gültigkeit beider Lehren vollkommen überzeugt, ich glaube nicht bloss an Neubildung von Formen durch direkte Anpassung und an solche durch Selektion, sondern jch möchte meiner Meinung dahin Ausdruck geben, dass vielfach beide Modalitäten kombiniert den von uns beobachteten Effekt liefern.

Wenn ich heute mithin für die lamarckistische Anschaung eintrete, so geschieht es nicht, um damit gegen die Selektionslehre Stellung zu nehmen, sondern deshalb, weil von beiden Lehren gerade die lamarekistische heute noch viele Gegner und nur wenige überzeugte Anhänger hat.

Vielfach werden heute die Ansichten der Lamarckisten durch die Einführung der Bezeichnung "Neo-Lamarckismus" in einen gewissen Gegensatz zur Lehre Lamarck's selbst gebracht; es hat dies seinen Grund nicht nur darin, dass wir, die wir über die physiologischen und morphologischen Erfahrungen eines ganzen Jahrhunderts verfügen, die einschlägigen Probleme anders und, wie wir glauben, ihrem Wesen näher tretend betrachten; es er- 
scheint dies auch begründet durch die Geschichte der I.ehre. Lamarck begründete seine Anschauungen \%u einer \%eit, in welcher die Descendenztheoric erst um ihre Anerkennung ringen musste; der Kampf um diese Hauptfrage, der einem Kampfe zwischen verschiedenen Weltanschauungen gleichkam, liess volles Verständnis für eine descendenztheoretische Nebenfrage nicht aufkommen. Und als dann 50 Jahre später das Auftreten Darwin's und seiner Anhänger der Descendenztheorie zu einem in der (ieschichte der Wissenschaft einzig dastehenden Siegre verhalf, da war es gan\% natürlich, dass die Anhänger der neuen Lehre sie z.unächst als mit der Selektionstheorie unzertrennlich verbunden betrachteten.

Nicht in der Absicht, dic alten Anschaumgen Lamarck's wieder zur Geltung zu bringen, sondern vollständig unahhängig, aus der darwinistischen Betrachtungsweise heraus entwickelte sich allmählich wieder mit zwingender Gewalt die Jeber\%eugung, dass neben dem Selektionsprinzip in der organischen $1 \mathrm{~T}$ elt noch ein zweites umgestaltend wirkt.

Heute können wir wohl sagen, dass unter den Butanikern lamarckistische Anschaungen immer mehr an Verbreitung gewinnen ${ }^{20}$ ), dass auch Paläontologen - gerade der lehrreiche Vortrag Koken's im Vorjahre hat das wieder bewiesen ${ }^{21}$ ) - die Berechtigung derselben anerkennen; wenn auf zoologischer Seite heute noch vielfach die Ueberzugung von der Allmacht des Selektionsprinzipes herrscht, so ist dies nicht bloss auf die Macht der wissenschaftlichen Autorität zurückzuführen, sondern zum guten Teile darauf, dass gerade in den hier in Betracht kommenden Eigentümlichkeiten die beiden Erscheinungskomplexe des Lcbens, das Tierreich und die Pflanzenwelt, sich vielfach verschieden zu verhalten scheinen.

Lassen Sie mich nun zu einer kurzen Darlegung unserer lamarckistischen Anschaungen und zur Andeutung der Beweise für die Zulässigkeit derselben übergehen. 
Ein Ueberblick über die Eigentümlichkeiten, welche Organismen im Laufe der phylogenetischen Entwickelung annehmen, zeigt uns alsbald die recht verschiedene Natur dieser Eigentümlichkeiten. Wir sehen einerseits Eigenschaften, welche mit Anpassungen an bestimmte Verhältnisse, unter denen der Organismus lebt, direkt nichts zu thun haben, welche insbesondere die Stellung des Organismus in der Stufenleiter der Formen, seine Organisationshöhe charakterisieren, wir nennen sie Organisationsmerkmale 22); wir beobachten andererseits Eigentümlichkeiten, durch welche sich vielfach Organismen derselben Organisationshöhe unterscheiden und die sich als Anpassungen an bestimmte Faktoren erkennen lassen: An passungsmerkmale. Ob die Teile einer Blüte in der Zahl 4 oder 5 vorhanden sind, ob die Samenanlagen den Rändern oder den Flächen der Fruchtblätter aufgewachsen sind, ob die I aubblätter der Anlage nach gegenständig oder wechselständig sind, das hat mit zweckmässigen Anpassungen zunächst nichts zu thun; es sind dies Organisationsmerkmale; dagegen werden wir in den Farben, Grössen und Stellungen der Blumenblätter, in der Ausbildung der Früchte, in Form und Grösse, Richtung und Behaarung der Laubblätter zahlreiche Anpassungsmerkmale nachweisen können. Organisationsmerkmale werden mit grosser Zähigkeit erblich festgehalten; Anpassungsmerkmale sind raschem Wechsel unterworfen.

Wenn wir uns nun die Frage vorlegen, wodurch eine Aenderung einer Eigentümlichkeit, und das ist ja die Entstehung einer neuen form, herbeigeführt werden kann, so ist es unschwer zu erkennen, dass wir die beiden Merkmalgruppen getrennt behandeln müssen; ein Merkmal, welches mit den momentanen Verhältnissen, unter denen der Organismus lebt, direkt nichts zu tun hat, wird durch andere Einwirkungen verändert werden, als ein Merkmal, das sofort bei Aenderung der Lebensbedingungen eine Aenderung erfahren muss, wenn nicht der Organismus existenzunfähig werden soll. 
Die Mutationslehre, welche $H$. de Vries ${ }^{23}$ ) vor einem Jahre in dieser Versammlung eingehend begründete, giebt uns ein Mittel an die Hand, um die Aenderung ron Organisationsmerkmalen mit oder ohne Zuhilfenahme der Selektion zu erklären. In der Kreuzung erblicken wir ein zweites Mittel, das denselben Effekt liefert und doch werden wir mit beiden Hilfsmitteln das Auslangen nicht finden ${ }^{2 \cdot}$ ). Beide Vorgänge machen die Mannigfaltigkeit der Organismenwelt verständlich, aber nicht die \%unehmende Komplikation der Organisation, die uns als Eortschritt in der Entwickelung erscheint. Dieser Fortschritt steht mit fortgesetzten Anpassungen an neue Funktionen und neue Lebensbedingungen in einem unverkennbaren Zusammenhange land darum können wir vielleicht von einer Erkenntnis in Bezug auf die Anpassungsmerkmale Aufklärung erwarten.

Gerade in Hinblick auf die Anpassungsmerkmale gehen Lamarckismus und Darwinismus wesentlich auseinander, ja, die Unnmöglichkeit, alle Anpassungsmerkmale auf dem Wege der Selektion zu erklären, hat den Neo-Lamarckismus hervorgerufen. Ich muss es für meine Person gestehen, dass eingehende monographische Studien ${ }^{25}$ ), die ich unternahm in der Absicht, konkrete Beweise für die Erwerbung von Anpassungsmerkmalen durch Selektion zu erlangen, mich allmählich immer mehr und immer zwingender zur Ueberzeugung brachten, dass in den untersuchten Fällen der Selektion eine nur untergeordnete Bedeutung zukommt.

Erwerbung von Anpassungsmerkmalen durch Variation und Selektion kommt bei Pflanzen gewiss vor; Erwerbung von Anpassungsmerkmalen durch Mutation oder Kreuzung mit oder ohne Selektion halte ich für möglich; die grösste Rolle dürfte aber hierbei - wenigstens im Pflanzenreiche - sogenannte „, direkte Anpassung“ spielen.

Unter direkter An passung ${ }^{2(i)}$ versteht der Lamarckismus die Fähigkeit der Individuen unter den jeweilig 
herrschenden Verhältnissen $z$ weckmässige Veränderungen zu erfahren und diesoerworbenen Eigentümlichkeiten zu vererben.

Lassen Sie mich die beiden in dieser Erklärung enthaltenen Voraussetzungen des Lamarckismus kurz behandeln.

Es wird kaum nötig sein, die Anpassungsfähigkeit der pflanzlichen und tierischen Individuen eingehend zu besprechen. Sie ist eine Tatsache, die wir nicht nur bei all' unseren Untersuchungen, sondern auch im täglichen Leben, an uns selbst konstatieren können; sie wird auch von den Gegnern lamarckistischer Anschauungen durchaus nicht geleugnet.

Wenn wir Pflanzen aus der alpinen Region in die Ebene, aus klimatisch begünstigten Gebieten in rauhere Gegenden, ja von einem Standorte auf einen zweiten verpflanzen, so ändern sie ihr Aussehen in einer Art und Weise, dass wir den direkten Zusammenhang mit den bedingenden Faktoren der Umgebung: ganz gut erkennen.

Es ist dies niemals in exakterer Weise bewiesen worden als durch Bonnier ${ }^{27}$ ), der Pflanzenstöcke teilte, die Teilstücke verschiedenen Kulturbedingungen aussetzte und das individuell verschiedene Verhalten der so gewonnenen Pflanzen beobachtete. Es wird also, soweit es sich um die Anpassungsfähigkeit der Individuen handelt, nicht so sehr der Nachweis der Erscheinung selbst notwendig sein, als vielmehr die Feststellung einiger Begleiterscheinungen, welche vielleicht geeignet sind, auf das Wesen der Vorgänge ein Licht zu werfen.

Da ist vor allem zu konstatieren, dass durch direkte individuelle Anpassung niemals, soweit unsere Erfahrungen reichen, etwas absolut Neues in Erscheinung tritt, sundern nur Modifikationen oder Umgestaltungen schon vorhandener Eigentümlichkeiten bewirkt werden. In dieser Hinsicht stellt die direkte Anpassung geradezu einen Gegensatz zur Veränderung durch Mutation dar. 
Weiter ist es von Wichtigkeit, hervorzuheben, dass durch direkte Anpassung selten nur eine Eiggentümlichkeit verändert wird, sondern dass sich zumeist Eigenschaften so mit anderen kombiniert erweisen, dass auch nicht direkte betroffene eine Mitveränderung crleiden.

Schliesslich erscheint es nicht unwichtig, zu bemerken, dass Individuen verschiedener Arten sich hinsichtlich der indiriduellen Anpassungsfähigkeit recht verschieden verhalten; manche besitzen in stauneneregendem Masse die Fähigkeit, sich den rerschiedensten l.ebensbedingungen zu accomodieren; andere charakterisiert eine gewisse Starrheit der physiologischen und morphologischen Ausbildung, welche eine Anpassung geradezu ausschliesst.

Es ist wichtig, diese drei Begleiterscheinungon der individuellen Anpassung festzustellen, da - wie wir sehen werden dieselben Begleiterscheinungen bei der erblichen Erwerbung ron Anpassungsmerkmalen sich beobachten lassen; ein Umstand, der allein schon auf einen innigen Zusammenhang beider Vorgänge hindeutet.

Dic erste Voraussetzung des Lamarckismus, die individuelle Anpassungsfähigkeit, kann ich als feststehend betrachten. Viel mehr umstritten ist die zweite Voraussetzung des Jamarckismus, die Fähigkeit der Vererbung der durch direkte Anpassung erworbenen Eigenschaften; um diese Voraussetzung dreht sich der Kampf für und wider diese Lehre. Auf der einen Seite haben wir Forscher, welche die Vererbung erworbener Eigenschaften als über alle Zweifel festgestellt anschen; auf der anderen Seite stehen nicht wenige Vertreter der entgegengesetzten Anschauung, für welche ein solche Vererbbarkeit „noch in keinem einzigen Falle bewiesen wurde“.

Ich nehme in einem gewissen Sinne eine Mittelstellung ein; ich bin ron der Möglichkeit der Vererbung erworbener Eigenschaften - allerdings mit einer ganz bestimmten Erklärung dieser 
Bezeichnung - vollständig überzeugt; ich gebe aber gerne zu, dass das bisher vorgebrachte Beweismaterial vielfach nicht danach angethan ist, Gegner zu bekehren.

Vor allem muss ich hervorheben, dass es sich bei der uns hier beschäftigenden Frage natürlich nur um die Vererbung von durch direkte Anpassung erworbenen Eigenschaften handeln kann, nicht um Eigenschaften schlechtweg. Eine Verstümmelung, eine Organisationsstörung irgend welcher Art ist keine durch Anpassung erworbene Eigentümlichkeit. Ich kann darum den iange Zeit fortgeführten Streit um die Möglichkeit der Vererbung von Verstümmelungen, von durch Krankheiten hervorgerufenen Veränderungen im Hinblick auf unser Thema nicht ganz begreifen und wenn von seiten der extremen Vertreter der Selektionslehre die Vererbbarkeit solcher Veränderungen mit Erfolg geleugnet wird, so ist damit ein recht unglückliches Argument beseitigt; die Frage nach der Vererbbarkeit der durch direkte Anpassung erworbenen Eigentümlichkeiten ist damit in keiner Weise beantwortet.

Lassen Sie mich im folgenden einige Erfahrungen aus meinem Wissensgebiete anführen, welche das Zutreffen dieser zweiten Voraussetzung des Lamarckismus erweisen oder wenigstens als sehr wahrscheinlich erscheinen lassen dürften. Zunächst ein paar indirekte Belege, deren Wert aber durch die Bezeichnung „indirekt" nicht abgeschwächt werden soll.

Zahlreiche eingehende monographische Bearbeitungen von Pflanzengattungen der neuesten Zeit ${ }^{28}$ ) haben mit voller Sicherheit ergeben, dass in jüngster Zeit entstandene Arten in ihrer Verbreitung eine Gesetzmässigkeit insofern erkennen lassen, als sie in sich gegenseitig ausschliessenden Arealen vorkommen. Die Anpassung an die verschiedenen Lebensbedingungen dieser Areale kommt darin klar zum Ausdrucke. Die Thatsache an und für sich ist ja auch auf zoologischem Gebiete längst bekannt, sie bildete die berechtigte Basis einer eigenen A.bstammungslehre, 
der Wagner'schen Migrationstheorie ${ }^{29}$ ), die allerdings in ihren Schlüssen als nicht zutreffend bezeichnet werden muss. Ich habe auf die grosse Verbreitung der Erscheinung und deren Bedeutung für die praktische Systematik gelegentlich der Naturforscherversammlung in Nürnberg hingewiesen ${ }^{30}$ ). Die für uns wesentlichste Begleiterscheinung ist aber die, dass die vicariierenden Arten an den Grenzen ihrer Areale stets durch zahlreiche Uebergangsformen nicht hybrider Art miteinander verbunden sind, welche in ihren Merkmalen ebenso erbliche Konstanz zeigen.

Diese Konstatierung scheint in striktem Gegensat\% zu der Behauptung Hugo de Vries' zu stehen, welcher die Konstan\%. der Arten als "Beobachtungstatsache“"31) bezeichnete. Der Widerspruch ist ein scheinbarer. Es giebt Arten, die ohne Uebergangsformen sprungweise zu anderen werden und auf solche bezogen sich die de Vries'schen Untersuchungen; es, giebt aber auch Arten, welche allmählich in andere übergehen. Die Mannigfaltigkeit der Ursachen der Artbildung kommt gerade in diesem Umstande auf das Klarste zum Ausdrucke.

Es wäre vollkommen gezwungen, anzunehmen, dass überall in den Zwischenzonen zwischen zwei vicariierenden Arten und diese Zwischenzonen sind gerade dann, wenn eine der beiden Arten in zerstückten Arealen sich findet, ausserordentlich ausgedehnt*) - sich der Uebergang von einer Art in die andere unzähligemal durch zufällige, aber gleiche Variation und Selektion gleichsinnig wiederholte; die einzige, mir ungezwungen erscheinende Erklärung der Thatsache ist die, dass bei dem Vordringen einer Art aus einem Gebiete in das andere oder bei Aenderung der Beschaffenheit eines Teiles des ursprünglichen Areales direkt eine entsprechende Umprägung der Form eintrat.

*) Dies trifft besonders dann zu, wenn eine Art in der Höhenregion der Gebirge eine verwandte Thaiform vertritt; in diesem Falle lassen sich auf Hunderten von Bergen solche Uebergangsformen lionstatieren; vergl. Ber. d. deutsch. botan. Ges. 1900, S. (197). 
Nicht minder beweisend für die Existenz der direkten Anpassung und der Vererbung erworbener Eigenschaften erscheint mir das Vorkommen ernährungsphysiologischer Rassen oder Arten. Wir kennen zahlreiche Pflanzen, die nur auf Böden von bestimmter chemischer und physiologischer Beschaffenheit vorkommen und auf Böden von abweichender Natur durch ihnen sehr nahe stehende, aber von ihnen verschiedene Arten vertreten werden $\left.{ }^{32}\right)$. „Kalk“und "Kieselformen“ derselben Typen sind bekannte Beispiele. Besonders scharf tritt dieser Zusammenhang zwischen Ernährung und Ausbildung bei Parasiten hervor. Viele parasitäre Gattungen der Blütenpflanzen gehören zu den vielgestaltigsten (Orobanche, Cuscuta u. a.) und die Feststelling ernährungsphysiologischer Rassen bei parasitären Pilzen ${ }^{33}$ ) zählt zu den wichtigsten Erfolgen der modernen Mykologie. Es heisst doch dem Zufall eine zu grosse Rolle zuschreiben, wenn man annehmen will, dass einzelne Individuen zufällig die Fähigkeit besassen, sich anders zu ernähren und dann durch Selektion zu neuen Rassen führten! Ist es da nicht viel natürlicher, anzunehmen, dass die Fähigkeit, unter geänderten Ernährungsbedingungen zu leben, allmählich erworben und vererbt wurde und in morphologischen Veränderungen dann zum Ausdrucke kam? Die Frage wird bejahend beantwortet werden müssen, wenn, wie aus dem folgenden hervorgehen wird, in Betracht gezogen wird, dass analoge Vorgänge direkt schon beobachtet wurden.

Einen deutlichen Hinweis auf direkte Anpassung und Vererbung erworbener Eigenschaften sehe ich in vielen sogenannten Konvergenzerscheinungen, insofern sie Anpassungen an analoge Lebensverhältnisse darstellen. Die Floren der Meeresküsten, der sandigen Dünen, der felsigen Hochgebirgsgipfel zeigen auf der ganzen Erde analoge Beschaffenheit. Pflanzen der allerverschiedensten systematischen Zugehörigkeit liefern analoge Anpassungsformen. Wenn an der oberen Grenze der Wälder zwischen diese und die baumlose Hochgebirgsregion auf der ganzen Erde ein 
Gürtel eigentümlich angepasster IIolzpflanzen sich einschaltet, der bald aus Arten der Gattung Pinus, bald aus Erlen oder Birken, aus Rhododendron-Arten oder gar aus Bambusen besteht, - wenn überall auf der Erde, wo klimatische Faktoren es erfordern, bei Tausenden von Pflanzenarten die, eine zweckmässige Anpassung darstellende, Umbildung ausdauernder, sog. ,immergrüner" Blätter in ,sommergrüne" sich vollzieht, solien wir dies einem an Tausenden von Orten in gleicher Weise wirkenden Einflusse des Zufalles und der Zuchtwahl zuschreiben oder ist es nicht viel natürlicher, an eine überall in analogen Bildungen zum Ausdrucke kommende direkte Wirkung analoger Lebensbedingungen zu denken?

Als ein Beweis für die Vererbung erworbener Eigentümlichkeiten ist von vielen Seiten schon die Tatsache geltend gemacht worden, dass Nichtgebrauch eine Reduktion von (Organen zur Folge hat. Es ist ron Weismann der Versuch gemacht worden, auch diesen Vorgang mit Zuhilfenahme der Selektionslehre zu erklären und insbesondere den Begriff der Cremminalselektion hier in Anwendung zu bringen ${ }^{31}$ ). Ich kann nicht sagen, dass auf mich Weismann's geistreiche Ausführungen in diesen Punkte überzeugend wirkten ${ }^{35}$ ). Dic einzige natürliche Erklärung der Erscheinung, dass nicht benutzte Organe allmählich sich zurückbilden, bietet der Lamarckismus. Funktion befördert die Ausbildung, Wegfall oder Einschränkung der Funktion bedingt die Reduktion eines Organes am Individuum; allmähliche Reduktion im Laufe phylogenetischer Entwickelung ist nur durch Verbindung dieses Vorganges mit erblicher Uebertragung der erworbenen Eigentümlichkeit zu verstehen. Wir kennen auf botanischem Gebiete einige lehrreiche Beispiele für eine derartige Reduktion durch Nichtgebrauch ${ }^{36}$ ). Unsere Getreidearten und unsere Fenerbohne stammen von ausdauernden Pflanzen ab. Die durch Jahrtausende, respektive Jahrhunderte eingetretene Unmöglichkeit, von den Organen zum Ueberwintern Gebrauch zu machen, hat eine 
vollständige Rückbildung derselben zur Folge gehabt. Von der Einwirkung einer Selektion kann hier nicht die Rede sein.

Besondere Bedeutung möchte ich einigen direkten Beweisen für die Vererbung der durch direkte A npassung erworbenen Eigentümlichkeiten zuschreiben. Auch solche existieren. Wir werden sie begreiflicherweise zunächst Arbeitsgebieten entnehmen. für welche die kontinuierliche Züchtung von Organismen und die genaue Kontrolle der Züchtungsergebnisse von grösster Bedeutung ist und solche Arbeitsgebiete sind einerseits das Studium der Mikroorganismen, andererseits die land- und forstwirtschaftliche Botanik. Mikroorganismen sind überdies dadurch zur Klärung einschlägiger Fragen von besonderem Werte, weil sie die Möglichkeit liefern, in kurzer Zeit zahlreiche Generationen zu erhalten, so dass an ihnen speziell die Frage der Vererbung von im Laufe der Generationen erworbenen Eigentümlichkeiten studiert werden kann ${ }^{37}$ ).

Seit der für die ganze Bakteriologie bedeutungsvoll gewordenen Entdeckung Pasteur's (1879), dass alte Kulturen des Hühnercholera-Bakteriums, die längere Zeit unter dem Einflusse des Sauerstoffzutrittes standen, ihre Virulenz verlieren, sind Untersuchungen über die Variabilität der Spaltpilze infolge von geänderten Kulturbedingungen in grosser Zahl durchgeführt worden ${ }^{35}$ ). Sie haben das übereinstimmende Resultat ergeben, dass es bei solchen gelingt, durch fortgesetzte, zahlreiche Generationen umfassende Kultur unter bestimmten Vegetationsbedingungen Eigentümlichkeiten hervorzurufen, die erblich festgehalten und erst wieder infolge eines neuen analogen Anpassungsvorganges abgestreift werden. Diese Eigentümlichkeiten sind nicht etwa geringe morphologische Abweichungen, sondern vor allem wird das physiologische Verhalten geändert, was auf tiefgehende Beeinflussung zurückschliessen lässt. Aenderungen im ernährenden Substrate, in der Einwirkung von Wärme, licht und Sauerstoff sind die verursachenden Faktoren, die in der Aenderung der Beschaffen- 
heit der Stoffwechselprodukte, der Wirkung auf das Substrat, der Art der Fortpflanzung zur Geltung kommen. Wenn auch nicht alle einschlägigen Versuche beweiskräftig sind infolge des Umstandes, als nicht immer das Mitwirken der Selektion ausgeschlossen wurde, so kann es doch keinem Zweifel unterliegen, dass Formneubildung durch direkte Anpassung bei Bakteriaceen möglich ist, weshalb auch die angewandte Bakteriologie heute bekanntlich schon mit grösstem Erfolge sich auf diese Tatsache stiutzt.

Ausserordentlich interessant und in theoretischer wie praktischer Hinsicht bedeutungsvoll sind die Untersuchungen E. Ch. Hansen's über Hefen ${ }^{39}$ ). Er gelangte bei seinen umfassenden Studien zu dem Ergebnisse, dass bei einzelnen Hefepilzen, also Arten der Gattung Saccharomyces, es gelingt, bei fortgesetzter Kultur unter abnorm hohen Temperaturen die Sporenbildung, eine Art der Fortpflanzung, zu unterdrücken und auf diese Weise asporogene Rassen zu züchten, welche zunächst auch unter normalen Kulturbedingungen asporogen bleiben. Die Experimente wurden durch I2 Jahre mit vielen Hunderten von Generationen durchgeführt und sind für die Entscheidung der hier behandelten Frage von um so grösserer Bedeutung, als zu ihrem Ausgangspunkte eine isolierte Zelle gewählt wurde. Selektion spielte hier keine Rolle.

Eine Reihe von Untersuchungen ergab die Möglichkeit, höher stehende, also vor allem vielzellige Pilze allmählich an neue Ernährungsbedingungen $z u$ accomodieren und sie zum erblichen Festhalten dieser neu erworbenen Ernährungsweise zu bringen. Ray ${ }^{40}$ ) hat dies für Aspergillus albus gezeigt, Hunger und Errera ${ }^{41}$ ) für Aspergillus niger.

Doch auch über Blütenpflanzen liegen Erfahrungen vor.

Eine vielfach bestätigte Thatsache, welche landwirtschaftlich und nationalökonomisch von grosser Wichtigkeit ist, ist die, dass auch bei manchen Kulturpflanzen sich die Anpassung an 
regionale Verschiedenheiten deutlich zeigt und der Mensch trotz aller Bemühungen nicht im Stande ist, ihr für die Dauer wirksam entgegenzutreten ${ }^{42}$ ).

Ungarn hat seine charakteristischen Weizensorten, ebenso Mähren und andere Gebiete Europas. Versuche, in diese Gebiete gewisse Weizensorten aus anderen Ländern wegen ihres besonderen Wertes einzuführen, haben wenig Erfolg; dieselben nähern sich immer wieder langsam, aber unaufhaltsam der Beschaffenheit der heimischen Sorten. In diesem Falle sehen wir einen Kampf zwischen dem Selektionsprinzipe und der direkten Anpassung, in welchen der Mensch zu Gunsten des ersteren eingreift; der Kampf endet trotzdem mit dem Siege der direkten Anpassung. Schübeler ${ }^{43}$ ) hat die erwähnte Erscheinung experimentell geprüft und ist zu dem Resultat gekommen, dass, wenn man in Skandinavien Getreidearten aus den Niederungen in Gebirgsgegenden bringt, sie daselbst trotz geringerer Mitteltemperaturen in kürzerer Zeit ihre Entwickelung abschliessen und diese erworbene Eigentümlichkeit auch dann noch eine Zeit lang festhalten, wenn sie neuerdings in der Ebene gezogen werden.

Zu ganz gleichen Resultaten gelange ich bei experimentellen Untersuchungen, die ich seit sechs Jahren mit einer anderen Kulturpflanze, mit dem Lein durchführe. Diese Untersuchung ist noch nicht abgeschlossen, doch darf ich heute schon einige sicher stehende Resultate erwähnen. Wenn wir den Lein derselben Rasse in klimatisch verschiedenen Gegenden untersuchen, so finden wir ihn in seiner Entwickelungsdauer und in einer Reihe morphologischer Eigentümlichkeiten den lokalen Verhältnissen angepasst. Je kürzer z. B. die Vegetationszeit des betreffenden Gebietes ist, desto rascher entwickelt sich die Pflanze. In anderen Gegenden aus Samen gezogen, nimmt die Pflanze nun durchaus nicht sofort die diesen neuen L.ebensbedingungen entsprechende 
Beschaffenheit an, sondern behält zunächst noch ihre Anpassung an die früheren Lebensbedingungen bei.

Ausserordentlich wertroll erscheinen mir die Untersuchungen Cieslar's $\left.{ }^{14}\right)$ über das Verhalten von Waldbäumen verschiedener Samenprovenienz. Bäume derselben Art zeigen bekanntlich in verschiedenen Höhenlagen unter verschiedenen L ebensbedingungen verschiedene Wachstumsverhältnisse; dies ist zum Teil der direkte Ausdruck der fördernden oder hemmenden Wirkung der Tebensbedingungen, zum Teil der Ausdruck der direkten Anpassungsfähigkeit. Es ist nun schr bemerkenswert. dass beispielswcisc Fichten und Lärchen, die aus alpinen Samen in der Ebene gezogen wurden, durch langsamen W'uchs und geringe Zuwachsgrössen die erworbenen Eigentümlichkeiten ihrer Vorfahren aufweisen.

Schliesslich möchte ich noch einer schönen Beobachtung gedenken, dic wir Jakowatzis) verdanken. (ielegentlich einer monographischen Untersuchung der grossblütigen Enziane der Alpen, welche unter dem Sammelnamen Gentiana acaulis bekannt sind, ergab sich, dass diese Enziane ciner ganzen Reihe sehr nahe verwandter, morphologisch deutlich verschiedener Arten angehören, welche sich in ancinander grenzenden Grebieten vertreten. Die Abhängigkeit der Artbildung von lokalen Einflüssen -ist deutlich zu sehen. Seit Jahrhunderten wird nun die Gientiana acaulis in Gärten als Zierde sngenannter \1penpflanzenanlagen gezogen und ist hier allmählich zu einer Pflanze gerrorden, die ron den wildwachsenden Arten morpholngisch stark abweicht. Künstliche Zuchtwahl hat gewiss dabei nicht mitgespielt, da die morphologische Abweichung den Züchtern infolge des Umstandes nicht bekannt wurde, dass sie die dekorative Wirkung der Pflanze nicht beeinflusst; aber auch natürliche Zuchtwahl ist wohl auszuschliessen, da, wie die Erfahrung zeigt, die spontanen Arten der Alpen kultiviert werden können. Es bleibt nichts anderes übrig, als anzunehmen, dass im laufe der Zeit unter 
den veränderten Ernährungsbedingungen und klimatischen Einflüssen in den Gärten die Pflanze in direkter Anpassung zu der heute uns entgegentretenden Gartenpflanze wurde.

Ich glaube nach dem Gesagten behaupten zu können, dass nicht nur die erste Voraussetzung des Lamarckismus, die Anpassungsfähigkeit des Individuums, sondern auch die zweite, die Fähigkeit des Organismus, die durch direkte Anpassung erworbenen Eigentümlichkeiten zu vererben, als zutreffend bezeichnet werden kann.

Ist dies der Fall, dann können wir auch den Lamarckismus als solchen für berechtigt halten, unabhängig davon, ob wir die von ihm angenommenen Vorgänge zu erklären vermögen oder nicht ${ }^{45}$ ). Ich betone dies mit Rücksicht auf den so oft gemachten Einwand, der Lamarckismus sei unannehmbar, weil die von ihm angenommenen Vorgänge nicht erklärt werden können; selbst wenn dies der Fall wäre, dürfte uns dies nicht abhalten, die offenkundigen Tatsachen anzuerkennen, um so weniger, als wir ja sehr gut wissen, wie oft in der Biologie sogenannte Erklärungen nichts anderes als Umschreibungen des zu Erklärenden sind.

Doch trifft der erwähnte Einwand gar nicht zu; wir sind in der Iage, uns einer Erklärung der beobachteten Phänomene stark zu nähern. Zunächst wollen wir zu diesem Zwecke einige Eigentümlichkeiten der Neubildung von Formen durch direkte Anpassung feststellen, die zum Verständnis der Vorgänge von Wichtigkeit sind.

Durch direkte Anpassung entsteht, soweit unsere Erfahrungen reichen, niemals sofort etwas vollständig Neues, sondern es treten Modifikationen der schon vorhandenen Eigentümlichkeiten auf. Ferner ist es wichtig, festzuhalten, dass länger währende individuelle Anpassung notwendig zu sein scheint, um erbliches Festhalten der erworbenen Eigentümlichkeiten zu ermöglichen*).

*) Eine dritte, S. I3, erwähnte Eigentümlichkeit, dass nicht alle Organismen in gleichem Masse anpassungsfähig sind, steht in voller Uebereinstimmung mit den An- 
Beide Erscheinungen stehen mit der Auffassung der direkten Anpassung als einer "funktionellen Anpassung“47) in vollem Einklange. Unter Angepasstsein eines Organes verstehen wir die Fähigkeit desselben, entsprechend zu funktionieren. In der Zunahme oder Erwerbung der Funktionsfähigkeit liegt mithin das Wesen der Anpassung. Bei einem Erklärungsversuch derselben handelt es sich also darum, ob die Veränderung rom Organe ausgeht und die Funktion die Folge dieser Veränderung ist oder ob die Funktion selbst das Organ entsprechend umgestaltet. In der verschiedenen Art der Beantwortung liegt der Unterschied zwischen Darwinismus und Jamarckismus; ersteres ist die Voraussetzung der Selektionslehre, letzteres die der Lehre von der direkten Anpassung.

Ich gebe zu, dass es vorkommen kann, dass zufällig eine Organisation entsteht, welche die Möglichkeit einer Funktion zur Folge hat und darum verhalte ich mich, wie schon gesagt, der Selektionstheorie gegenüber durchaus nicht ablehnend; ich bin aber davon überzeugt, dass in der Regel die Funktion die Ausbildung eines Organes beeinflusst. Diese Beeinflussung der Organe durch deren Funktion bahnt eine annähernde Erklärung für die individuelle Anpassungsfähigkeit, also für eine unserer Voraussetzungen an.

Die zweite Voraussetzung; die erbliche Festhaltung der erworbenen Anpassungsmerkmale, bedingt, dass die funktionelle Aenderung irgend eines Organes auch in der Qualität der Fortpflanzungszellen zum Ausdruck kommt. Für jene Fälle, in welchen die Fortpflanzungszellen von dem Anpassungsvorgange direkte betroffen werden, wie bei den Protisten, macht diese Voraussetzung keine Schwierigkeiten; wohl aber für die höher stehenden Organismen mit scharf differenzierten Fortpflanzungsorganen. Wenn wir nun

schauungen H. de Vries' ${ }^{48}$ ), der auch für Formueubildung durch Mutation M utationsperioden annimm. - Die Unfähigkeit extrem angepasster Formen, sich zu accomodieren, steht mit der Erscheinung des Aussterbens der Arten im Zusammenhang ${ }^{1 .}$ ). 
auch für diese Mitbeeinflussung dier Fortpflanzungsorgane durch die direkte Anpassung heute noch keine exakte Frkklärung haben, so kann sie uns doch nicht als etwas vollständig Unverständliches erscheinen, wenn wir an andere wohlbekannte, ähnliche biologische Vorgänge denken, die wir auch nicht erklären können, aber trotzdem als existierend betrachten müssen. Ich verweise beispielsweise auf die Erscheinung der Korrelation, welche sich darin äussert, dass Organe ganz verschiedener Ausbildung, welche morphologisch in gar keinem direkten Zusammenhange stehen physiologisch einen solchen Zusammenhang bekunden, welcher bewirkt, dass eine Veränderung des einen eine Umgestaltung des anderer: zur Folge hat. Gerade so, wie eine Veränderung in der Krone eines Baumes eine solche in der Wurzel korrelativ veranlasst, wie das Nichtfunktionieren von Blüten gelegentlich die Ausbildung von Axillarknospen an entfernten Stellen des Stammes zur Folge hat, ebenso ist die Beeinflussung der Fortpflanzungsorgane durch adaptive Veränderung somatischer Körperteile ganz gut denkbar.

Der Hinweis auf die Erscheinungen der Korrelation dürfte um so mehr hier am Platze sein, als - wie schon S. I3 hervorgehoben - die direkte Anpassung selten ein Organ allein betrifft, sondern fast immer mit korrelativen Vorgängen verbunden ist.

Schliesslich sei es mir noch gestattet, mit wenigen Worten auf die Bedeutung der Formneubildung durch direkte Anpassung für die Entwickelung der Organismenwelt überhaupt hinzuweisen. Ich habe früher betont, dass Mutation und Selektion, eventuell auch Kreuzung, zwar die Mannigfaltigkeit der Organismenwelt begreifen lassen, dass sie aber nicht ausreichen, um das Phänomen der allmählichen Steigerung der Organisationshöhe verstänảlich zu machen. In der direkten Anpassung haben wir einen Vorgang kennen gelernt, welcher, den Anforderungen des Lebens folgend, eine allmähliche Umgestaltung des Organismus herbeiführt, welcher entsprechend 
der Vervielfältigung der Lebensbedingungen eine Differenzierung der Organe bewirkt, dabei aber funktionell nicht beeinflusste Organe wenn auch nur reduziert oder latend - zulässt. Der Gedanke, dass durch unermessliche Zeiträume fortwirkende direkte Anpassung die allmählich steigende Organisationshöhe bewirkte, findet eine Bestärkung in der Erfahrung, dass ein guter Teil der Merkmale, die uns heute als Organisationsmerkmale entgegentreten, denn doch auf Anpassungen zurückführbar ist. Ich brauche da nur daran zu erinnern, dass der vielleicht wichtigste Abschnitt in der Entwickelung der Pflanzenwelt, der Uebergang der Thallophyten zu den Cormophyten durch Vermittelung der Muscineen, mit all' seinen morphologischphysiologischen Eigentümlichkeiten uns verständlich wird in dem Augenblicke, in dem wir allmähliche Anpassung der an das Wasserleben adaptierten Pflanzen an das Iandleben während dieses Entwickelungsabschnittes annehmen.

Ich sehe daron ab, die Tragweite der lamarckistischen Anschauungen für die verschiedensten Gebiete des menschlichen Tuns und Denkens zu erörtern. Es liegt nahe, von welch' grosser Eedeutung es ist, wenn nicht bloss die Begünstigung des an und für sich schon Passenden - wenn auch nur relativ Passenden durch Selektion einen Fortschritt ermöglicht, sondern wenn es daneben ein zweites Prinzip gicbt, nach dem allmählich, aber unaufhaltsam eine Beeinflussung der Gesammtheit der L,ebewesen stattfindet. Ich sehe ron einer diesbezüglichen Erörterung ab, weil es mir zunächst darum zu tun ist, an einer streng sachlichen und objektiven Begründung einer auch von mir vertretenen naturwissenschaftlichen Anschauung mitzuwirken. 


\section{Anmerkungen.}

I) Hertwig, O., Die Entwicklung der Biologie im iq. Jahrhundert. Verh. d. Ges. deutscher Naturf. u. Aerzte, igor. Leipzig (F. C. W. Vogel) und Jena (G. Fischer).

2) Ziegler, H. E., Ueber den derzeitigen Stand der Descendenzlehre in der Zoologie. Jena (G. Fischer) r 902.

3) Vries, H. de, Die Mutationen und die Mutationsperioden bei der Entstehung der Arten. Leipzig (Veit \& Co.) I 90 I.

4) Koken, E., Paläontologie und Descendenzlehre. Jena (G. Fischer) I 902.

5) Weismann, A., Vorträge über Descendenztheorie. 2 Bände. Jena (G. Fischer) I902.

6) Wettstein, R. v., Der gegenwärtige Stand unserer Kenntnisse betreffend (lie Neubildung von Formen im Pflanzenreiche. Ber. d. deutsch. bot. Ges., I900, Bd. XVIII. - Ueber direkte Anpassung. Almanach der Kaiserl. Aliademie d. Wissenschaften. Wien 1902.

7) Lamarck, J., Philosophie zoologique, I809.

8) Spencer, H., System der synthetischen Philosophie. Bd. II: Die Prinzipien der Biologie. Deutsche Uebers. von Vetter, 1876. I. Aufl. der engl. Ausgabe i 863 .

9) Eimer, Th.. Die Entstehung der Arten auf Grund von Vererben erworbener Eigenschaften nach den Gesetzen organischen Wachsens, I 888 - I 90 I.

Io) Naegeli, C., Mechanisch-physiologische Theorie der Abstammungslehre, I 884 .

I I) Kassuwitz, M., Allgemeine Biologie. 2 Bände. I899. Anscheinend ähnliche Gedanken, wie die der vorliegenden Schrift, enthält die Abhandlung von Costantin, J., „L’hérédité acquise, ses conséquences horticoles, agricoles et medicales. Collect. Scientia. Ser. biol,, No. I2. Leider konnte ich in dieselbe nicht mehr vor Drucklegung meiner Schrift Einsicht nehmen. 
I 2) Darwin, Ch., Ueber die Entstehung der Arten durch naturliche Zuchtwahl. I. engl. Ausgabe I 859.

I 3) Kölliker, Anatomisch-systematische Beschreibung der Alcyornarien. Frankfurt a. M. I 872.

I4) Roux, W., Der Kampf der Teile in Organismus, I88 I.

I 5) Vgl. insbesondere Weismann, A., Aufsätze über Vererbung und verwandte biologische Frägen, I 892. - Vorträige über Descendenztheorie, I 902 .

I6) Kerner, A., Pflanzenleben, I. Aufl., i 80 I, Bel. II.

I 7) Korschinsky, S., Heterogenesis und Erolution. Naturw. Wochenschr., I 899, Bd. XIV, S. 273; ferner in Flora, I 901 . Ergïnzungsband, S. 240.

I8) Vries, H. de, Die Mutationsthenrie. Leipzig Ioor.

I9) Wettstein, R. r., Monographie der Gattung Euphrasia. Leipzig I 895, S. 37. - Schriften d. Vereins zur Verbr. natuw. Kenntn. Wien I897, Bd. XXXVIl. - Die Arten der Gattung Gentiana, Sect. Endotricha und ihr entwickelungsgeschichtlicher \%usammenhang. Denkschr. d. Kais. Akademic Wien, I896. - Vgl, auch Anm. 6. - Vgl. ferner Göbel, K., Ueber Studium und Auffassung der Anpassungserscheinungen bei Pflanzen. Festrede. München I8g8; Reinke, J., Gedanken über das Wesen der Organisation. Biol. Centralbl. XIX, No. 3/4, S. I 2 I; Klinge, J., in Acta horti P'etrop., Bd. XVII, Fasse. 2, I 899; Pfeffer, W., Pflanzenphysiolngie, 2. Aufl., II (1901); Wiesner, J., Biologie der Pflanzen, 2. Aufl., Igor; Solms-Laubach, H. in Bot. Zeitung I9OI, S. I67 ff.

20) Ausser den in Anm. I g genamten insbesondere VTaming, E., Henslow, G., Errera, L., Costantin, J., Focke, E. u. a.

2 I) Vgl. auch Suess, E., Abschiedsvorlesung, S. 2 (1902). - Stein mann, G., Palacontologie und Abstammungslehre an Ende des Jahrhunderts. Freiburg i. B. (Lehmann), I 899.

22) = „Spezifische Merkmale“, „morphologische Merkmale“ verschiedener Autoren. Es ist ein Verdienst Naegeli's, auf den prinzipiellen Unterschied zwischen beiden Merkmalsgruppen nachdrücklich hingewiesen zu haben. Mechan.-physiol. Theoric der Abstammungslehre, 1884.

23) Vgl. Anm. I 8.

24) Ueber den Anteil verschiedener Vorginge bei der Bildung neuer Formen im Pflanzenreiche vgl. mein Sammelreferat in Rer. d. deutsch. bot. Ges., I 900, Bd. XVIII.

25) Vgl. Anm. I 9. 
26) Nahezu gleichbedeutend mit ,direkter Anpassung" sind folgende Ausdrüclie: „,direkte Ausgleichung“ resp. „Direct Equilibration“ (Spen cer), „Selbstregulierung“" (Warming), ,Artbildung durch direkte Bewirkung“( $\mathrm{N}$ a egeli), ,Artbildung durch Korrelation“(Wettstein). Die Bezeichnung ,direkte Anpassung “ ist nicht gerade die glücklichste, da nicht alle durch direkten Einfluss der Ungebung hervorgerufenen Abweichungen Anpassungen sind; wenn ich den Ausdruck hier mit dieser Einschränkung doch gebrauche, so geschieht es insbesondere, weil ich nicht durch Gebrauch eines ungeläufigen Ausdruckes die Darstellung unverständlicher machen möchte.

27) Bonnier, G., Cultures experimentales dans les Alpes et les Pyrenees. Rer. gen. de Bot., II, p. 5 I 3 ss.

28) Vgl. das Verzeichnis in Anm. I 9 zu Wettstein, R. v., Ueber direkte Anpassung. Wien I 902.

29) Wagner, M., Gesammelte Aufsätze. Basel I 889.

3o) Wettstein, R. v., Die geographische und systematische Anordnung der Pflanzenarten. Verh. d. Ges. deutsch. Naturf. u. Aerzte. Nürnberg I 893. - Grundzüge der geographisch-morphologischen Methode der botanischen Systematik. Jend (G. Fischer) I 898.

31) Die Mutationen und die Mutationsperioden, igor, S. 8.

32) Ein par Beispiele sind genannt in Ber. d. deutsch. bot. Ges., I 900 , S. ( I 97 ).

33) Vgl. Erikson, J., Ueber die Spezialisierung des Parasitismus bei den Getreiderostpilzen. Ber. d. deutsch. bot. Ges., XII, S. 295. Ueber weitere Iitteratur rgl. Ber. d. deutsch. bot. Ges., I goo, S. (I97).

34) Es ist hier nicht der Ort, $11 m$ ausführlicher auf die Frage einzugehen. Ich will nur folgende Bemerkungen einschalten. Weismann's Geminalselektion beruht auf der Annahme, dass die Determinanten des Keimplasmas in rerschiedener Weise ernährt werden und dadurch Gelegenheit zum Eingreifen der Selektion geboten ist. Mir will scheinen, dass damit die Frage, ob die darwinistische oder die lamarckistische Auffassung berechtigt ist, nur verschoben, aber nicht beantwortet wurde. Es wircl sich darum handeln, wodurch die verschiedene Ernährung der Determinanten veranlasst wird; ist die Verschiedenheit eine zufällige, dann allerdings ist die darwinistische, Weismann's Ansicht die richtige; ist die Verschiedenheit bedingt durch äussere Einflüsse direkt oder indirekt - damn ist 'gerade diese Annahme verschieden ernährter Determinanten des Keimplasmas eine Stütze des Lamarckismus. 35) Weismann, A., Vorträge, S. I $29 \mathrm{ff}$ 
36) Batalin, A., Das Perennieren des Roggens. Acta horti Petrop. XI, I89o. - Wettstein, R. v., Die Innovationsverhältnisse von Phaseolus coccineus. Oest. bot. Zeitschr., I 897 , S. 424.

37) Es darf allerdings auch nicht verschwiegen werclen, dass der Wert der mit Mikroorganismen er\%iclten Resultate deshall) ange\%weifelt wurde, weil ihre Vermehrung auf Teilung vegretativer Zellen beruht, so (lass erbliches Festhalten enworbener Eirgenschiften hier leichter verständlich ist, als bei höheren l'flanzen, bei welehen die durch Anpassung direkte veränderte Zelle nicht \%ugleich \%um Ausgangspunkt für ein nenes Individum wirl. Die Bedeutung der Versuche mit Mikroorganismen sehe ich dirin, disss sie deutlich eine daucrude Umstinmmung eines Organismus durch dielite Ampassung zeigen und (lás ist schliesshich das Wesentliche. Wie bei höheren Pfinzen die Unstinmung von dem beteiligten Teile auf die Fortpflanzungs\%ellen übertragen wird, las ist eine zweite Frage.

38) Siehe cie Litteratur\%usimmmenstellung in Ber. (l. (leutsih. lot. Ges. I900, S. (198), ferner in meiner mehrfach citierten Ablandlumg „Ueber direkte Anpassung", S. 25. - V Vgl. ferner Pfeffer, WV., l’flinzenphysiologie, 2. Aufl. (I gO I), II, S. 242.

39) Hansen, E. Clur., Om variationen hos ïljöstswampane oxh hos andra Saccharonyceter. Svenska Bryggare-füreningens Mänadsblal, I897; Ref. in Centralls. f. Balitoriol. u. P'arasitenk., II. Abt., Bal. IV', S. 89. - Neue Untersuchungen über die Sporenbildung bei Sarcharomyceten. Centralbl. f. Bakt., I899, II. Abt., Bel. V, S. I. - Mecldelelsen fra Carlsberg Laborat., I896, IV. Heft. - Compt. rend. d. Labor. d. Cartsberg, I goo, Bd. V, P. I.

40) Ray, J., Variations des chanpignons inferieurs sous l'influence du milieu. Rev. gen. (le Bot., I897, Tom. IX, p. I93.

4 I) Errera, L., Héréclité d'un caractère acquis chez un champignon pluricellulaire d'après les experiences le M. le Dr. Hunger. Bull. de l'Acad. roy. de Belg., I 899, No. 2, p. 8 I.

42) Nobbe, F., Handbuch der Samenkinde, S. 208 (I876). Schindler, F., Der Weizen in seinen Beziehungen zum Klima und das Gesetz der Korrelation. Berlin r 893 .

43) Schübeler, F. C., Viridarium Norvegicum. Norges Växtrige. Et Bidrag til Nord-Europas Natur og Kulturhistorie, 1885, Bd. I.

44) Cieslar, A., Die Zuchtwahl in der Forstwirtschaft. Centralbl. f. d. ges. Forstwesen, I 890. - Die Erblichkeit des Zuwachsvermögens 
bei den Waldbäumen. A. a. O. I 895. - - Neues aus dem Gebiete der forstlichen Zuchtwahl. A. a. O. I899.

45) Jakowatz, A., Die Arten der Gattung Gentiana; Sekt. Thylacites und ihr entwickelungsgeschichtlicher Zusammenhang. Sitzber. d. Wiener Akad. d. Wissensch., CVIII.

46) Vgl. selbst Weismann, A., Vorträge, Bd. II, S. 79: „Wenn sich zeigen liesse, dass grosse Gruppen von Erscheinungen sich auf keine andere Weise erklären liessen, als unter der Voraussetzung einer solchen Vererbung, so müssten wir dieselbe dennoch als wirklich annehmen, trotzdem es nicht beweisbar, ja nicht einmal theoretisch vorstellbar ist."

47) Soweit ich beurteilen kann, hat H. Spencer den Begriff der funktionellen Anpassung geschaffen. Vgl. Prinzip. der Biol., Deutsch. Ausg., Bd. I, S. 166 ff., S. 294 ff. u. a.

48) Vgl. Anm. 3 .

49) Weismann sagt (Vorträge, Bd. II, S. I 57): „Es ist das Unvermögen der Personalselektion, rascheren Wendungen der Lebensbedingungen zu folgen und excessive Bildungen in kurzer Zeit um ein Beträchtliches herabzusetzen", welches in solchen Fällen einer Art den Untergang bereitet. Ich möchte dem nicht in dieser allgemeinen Fassung zustimmen, da ja gerade Mutation und Personalselektion rasche Veränderung ermöglicht; es ist nach meiner Anschauung das Unvermögen extrem angepasster Folmen, sich rascheren Wendungen der Lebensbedingungen entsprechend direkte anzupassen, welches in solchen Fällen wohl zumeist den Untergang einer Art herbeiführt. 